Arq. Bras. Med. Vet. Zootec., v.68, n.2, p.431-438, 2016

\title{
Caracterização morfométrica de fêmeas, garanhões e castrados da raça Campolina baseada em índices
}

\author{
[Morphometric characterization of Campolina mares, stallions and \\ gelded horses using indexes] \\ J.E.C. Lucena ${ }^{1}$, S.A.B. Vianna ${ }^{2}$, F. Berbari Neto ${ }^{3}$, R.L.M. Sales Filho ${ }^{1,4}$, W.J.S. Diniz ${ }^{5}$ \\ ${ }^{1}$ Universidade Federal Rural de Pernambuco - UFRPE-UAG, Garanhuns, PE \\ ${ }^{2}$ Universidade Estadual do Norte Fluminense Darcy Ribeiro - UENF Campos dos Goytacazes, RJ \\ ${ }^{3}$ Universidade Federal do Espírito Santo - Ufes - Vitória, ES \\ ${ }^{4}$ Aluno de pós-graduação - Universidade Federal de Pernambuco - Recife, PE \\ ${ }^{5}$ Aluno de graduação - Universidade Federal Rural de Pernambuco - UAG - Garanhuns, PE
}

\begin{abstract}
RESUMO
O presente estudo objetivou avaliar os índices morfométricos para garanhões, machos castrados e fêmeas da raça Campolina, bem como caracterizá-los morfologicamente. Para tanto, foram utilizadas as medidas lineares de 4.840 garanhões, 19.037 fêmeas e 1.371 machos castrados, obtidas no banco de dados do Serviço de Registro Genealógico da ABCCCampolina, com base nas quais nove índices morfométricos foram calculados utilizando-se seis medidas lineares. A análise de variância foi realizada para avaliar o efeito do sexo e da idade sobre os índices obtidos. Verificou-se que ambos os fatores influenciaram os índices morfométricos. Garanhões apresentaram peso calculado aproximado superior tanto às fêmeas quanto aos machos castrados. De acordo com o índice peitoral, todas as categorias foram classificadas como "longe do chão". Em relação aos valores médios dos índices corporal e dáctilo torácico, garanhões e castrados foram classificados como mediolíneos e eumétricos, respectivamente, enquanto as fêmeas foram classificadas como brevilíneas e hipométricas. Conclui-se que os machos castrados e os garanhões da raça Campolina enquadram-se no padrão da raça para animais tipo sela, mas as fêmeas não. Entretanto, os índices de compacidade 1 e 2 apresentam resultados contraditórios e precisam ser melhor avaliados para fins de uso em classificações. Para a maioria dos índices, os animais castrados apresentaram-se melhor proporcionados que as demais categorias.
\end{abstract}

Palavras-chave: equinos, índices morfométricos, morfologia, seleção

\begin{abstract}
The present study aimed to evaluate the morphometric indexes for Campolina stallions, gelded and mare horses, as well as morphologically characterizing them. For this purpose linear measurements from 4.840 stallions, 19.037 mares and 1371 gelded horses were used, obtained from ABCCCampolina's stud book database, from which nine morphometric indexes were calculated from six linear measurements. Analysis of variance was carried out to evaluate the effect of sex and age on the indexes obtained. We found that both factors influenced the morphometric index. Stallions have presented higher estimate body weight values than mares and gelded horses. According to the pectoral index values all categories were classified as far from the ground. According to body index and dactyl thoracic index average values, stallions and gelded horses were classified as medium lined and middleweight, respectively, once the mares were classified as more compact and overweight. It is concluded that Campolina stallions and gelded horses fall within the breed standard for saddle animals, but mares don't. However, the compact indexes 1 and 2 showed contradictory results and need to be better evaluated for its intended use in classifications. For most indexes gelded animals showed better proportion than the other categories.
\end{abstract}

Keywords: horses, morphometric indexes, morphology, selection

Recebido em 23 de setembro de 2014

Aceito em 22 de setembro de 2015

E-mail: jorgelucena245@hotmail.com 


\section{INTRODUÇÃO}

A Campolina é uma raça de origem nacional de equinos marchadores, utilizada para sela, trabalho e lazer (ABCCCampolina, 1995), caracterizada por apresentar animais de elevado porte, robustez, resistência e andamento cômodo (Resende, 1979). O empirismo aplicado à seleção e a influência mercadológica exercida ao longo do tempo podem ter contribuído para a fixação de caracteres raciais em detrimento dos funcionais.

Sua funcionalidade pode estar baseada, entre outras particularidades, na harmonia das medidas e nas proporções lineares, que vão propiciar ao animal as habilidades necessárias ao andamento marchado (Berbari Neto, 2009). O estudo da morfometria é importante para a identificação morfológica, permitindo que a seleção seja feita em bases mais objetivas. Com base na análise morfométrica, as medidas corporais podem ser avaliadas em conjunto, tornando, assim, possível o desenvolvimento de critérios de seleção objetivos, em substituição às avaliações subjetivas frequentemente adotadas (Costa et al., 1998).

Segundo Ribeiro (1989), as medidas das diversas regiões do corpo do cavalo são úteis para cálculos de índices que permitem a apreciação das aptidões na escolha de espécimes destinados à reprodução e na seleção de diferentes tipos, tais como de sela, de corrida, de carga ou de tração. Lesbre (1920) estabeleceu para cavalos adultos de sela um sistema eclético de proporcionalidade no qual as medidas lineares devem manter determinadas relações com o comprimento da cabeça. Segundo as determinações do padrão da raça Campolina, que busca enquadrá-la no tipo sela, um animal adulto da raça deve ser mediolíneo e eumétrico. A altura de cernelha ideal de $1,62 \mathrm{~m}$ para os machos e de $1,56 \mathrm{~m}$ para as fêmeas evidencia que se procura um animal de porte alto, sem exagero (Inglês et al., 2004).

A utilização de índices que destaquem as relações entre as medidas de comprimento, de perímetro e de peso (Oom e Ferreira, 1987) no processo de seleção dos equinos ainda é pouco praticada no Brasil. Além disso, poucas pesquisas avaliando medidas lineares e índices morfométricos foram feitas, até o momento, com a raça Campolina. Esses estudos proporcionam relevantes informações técnicocientíficas com base nas quais a Associação Brasileira de Criadores de Cavalo Campolina (ABCCCampolina) pode direcionar suas estratégias para melhoramento da raça. Portanto, o presente estudo objetivou caracterizar e classificar morfologicamente, por meio de índices morfométricos, fêmeas, garanhões e castrados da raça Campolina, nascidos entre 1963 e 2006, registrados em definitivo em seus respectivos livros da ABCCCampolina.

\section{MATERIAL E MÉTODOS}

Os dados morfométricos utilizados neste estudo foram cedidos pela ABCCCampolina, dos quais foram extraídas informações referentes a 4.840 garanhões, nascidos entre os anos de 1963 e 2006, 19.037 fêmeas, nascidas entre os anos de 1964 e 2006, e 1.371 machos castrados, nascidos entre os anos de 1977 e 2006, com idades médias (em meses) de 51,49, 50,11 e 50,11, respectivamente, da categoria Puros de Origem, registrados em definitivo nos respectivos Livros CP5, CP6 e CP8.

As mensurações foram realizadas por 40 técnicos, todos habilitados e treinados pela ABCCCampolina, de modo a garantir a sua homogeneidade. As medidas lineares, realizadas segundo as descrições de Bretas et al. (2003), foram: altura na cernelha (ACER), altura no dorso (ADOR), altura dos costados (ACOS), comprimento de corpo (CCOR), perímetro de canela (PCAN) e perímetro torácico (PTOR). Com base nas medidas lineares, foram calculados os seguintes índices morfométricos, descritos e referendados por Oom e Ferreira (1987), Ribeiro (1989) e Torres e Jardim (1992): peso calculado aproximado (PCA), vazio subesternal (VSE), índice peitoral (IP), índice corporal (IC), índice dactilotorácico (IDT), índice de carga na canela (ICC), índice de conformação (ICF) e índice de compacidade (ICP), cujas fórmulas e classificações seguem abaixo:

$$
\begin{array}{ll}
» \quad & \mathbf{P C A}=\mathbf{P T O R}^{3} \times \mathbf{8 0}(\mathrm{kg}) \\
& \\
& \text { VSE }=\text { ADOR }-\mathbf{A C O S}(\mathrm{m}) \\
& \text { IP }=\text { ACOS }- \text { VSE }(\mathrm{m}) \\
& \mathrm{IP}>0 \Rightarrow \text { animal perto do chão } \\
& \text { IP }<0 \Rightarrow \text { animal longe do chão }
\end{array}
$$


$» \quad$ IC $=$ CCOR/PTOR (adimensional)

$\mathrm{IC}>90 \Rightarrow$ longilíneo

$0,86<\mathrm{IC}<0,89 \Rightarrow$ mediolíneo

IC $<0,85 \Rightarrow$ brevilíneo

» $\quad$ IDT $=$ PCAN/PTOR $($ adimensional $)$

IDT $>0,108 \Rightarrow$ hipermétrico

$0,105<$ IDT $<0,108 \Rightarrow$ eumétrico

IDT $<0,105 \Rightarrow$ brevilíneo

» ICC $=$ PCAN/PCA $(\mathrm{m} / \mathrm{kg})$

» $\quad \mathbf{I C F}=\mathbf{P T}^{2} / \mathbf{A C E R}$ (adimensional) sela

Valor de 2,1125 é ideal para o cavalo tipo

\section{$» \quad$ ICP1 $=$ PCA/ACER}

ICP1 $>3,15 \Rightarrow$ tipo tração pesada

ICP1 $>2,75 \Rightarrow$ tipo tração ligeira

ICP1 2,60 $\Rightarrow$ tipo sela

\section{ICP2 $=$ PCA/(ACER-100)}

ICP2 $>9,50 \Rightarrow$ tipo tração pesada

$8,00<\mathrm{ICP} 2<9,50 \Rightarrow$ tipo tração ligeira

$6,00<\mathrm{ICP} 2<7,75 \Rightarrow$ tipo sela

A análise de variância dos índices morfométricos foi realizada utilizando-se o software estatístico SPSS 22 para estimar os efeitos dos fatores sexo (comparando garanhões, machos castrados e fêmeas) e idade ao registro. Para esse fator, os animais foram classificados nas seguintes faixas etárias (em meses): faixa etária $1-36 \leq$ idade $\leq 48$; faixa etária $2-48<$ idade $\leq 60$; faixa etária 3 - idade $>60$.

Os dados foram testados quanto à homogeneidade das variâncias. Para as situações, os tamanhos das amostras testadas são muito grandes, o que permite que o pressuposto de normalidade possa ser relaxado, devido ao teorema do limite central (Bussab e Morettin, 2013). O teste de Levene foi aplicado a fim de verificar se o pressuposto de homogeneidade das variâncias era atendido (Levene, 1960). Nos casos em que tal pressuposto não foi atendido, como alternativa à ANOVA, ou seja, para os fatores sexo e idade das fêmeas, utilizou-se o teste de Kruskal-Wallis. Para garanhões e machos castrados, foi utilizada a ANOVA, exceto para IP e VSE, respectivamente.

\section{RESULTADOS E DISCUSSÃO}

Os valores médios dos índices obtidos dos animais avaliados no presente estudo são apresentados na Tab. 1. Para a maioria dos índices, o coeficiente de variação $(\mathrm{CV})$ foi menor ou próximo a $10 \%$ (Tab. 1). No entanto, o índice peitoral (IP) apresentou CV de $24,867 \%$ a $33,554 \%$. O IP é um índice que tem ligação com o posicionamento do centro de massas dos equinos, sendo importante para a otimização do equilíbrio corpóreo. Como as medidas utilizadas para o cálculo do IP apresentam baixo CV (altura de costado $=4,83$ e vazio subesternal $=$ 4,487), a variação pode estar relacionada com a falta de padronização na profundidade torácica dos animais da raça. Os elevados valores encontrados neste estudo seguem a tendência dos resultados encontrados por Berbari Neto (2005) e Campos (2006), os quais encontraram CV de 17,77 e $22,71 \%$ para garanhões e fêmeas da raça Campolina, respectivamente.

Tabela 1. Índices morfométricos de garanhões, machos castrados e fêmeas da raça Campolina

\begin{tabular}{|c|c|c|c|c|c|c|c|c|c|}
\hline \multirow{3}{*}{ Índice $^{1}$} & \multicolumn{9}{|c|}{ Categorias $^{2}$} \\
\hline & \multicolumn{3}{|l|}{ Garanhões } & \multicolumn{3}{|c|}{ Machos castrados } & \multicolumn{3}{|l|}{ Fêmeas } \\
\hline & $\bar{X}$ & $\mathrm{CV} \%$ & $\mathrm{n}$ & $\bar{X}$ & $\mathrm{CV} \%$ & $\mathrm{n}$ & $X$ & $\mathrm{CV} \%$ & $\mathrm{n}$ \\
\hline PCA & 499,635 & 11,699 & 4.814 & 481,646 & 11,368 & 1.367 & 492,712 & 13,299 & 18.991 \\
\hline VSE & 0,868 & 4,487 & 4.816 & 0,851 & 4,963 & 1.368 & 0,822 & 5,359 & 18.984 \\
\hline IP & $-0,218$ & 24,867 & 4.816 & $-0,204$ & 27,697 & 1.368 & $-0,172$ & 33,554 & 18.984 \\
\hline $\mathrm{IC}$ & 0,882 & 3,797 & 4.816 & 0,877 & 3,932 & 1.367 & 0,859 & 4,268 & 18.984 \\
\hline IDT & 0,106 & 6,101 & 4.816 & 0,107 & 5,952 & 1.366 & 0,103 & 5,595 & 18.971 \\
\hline $\mathrm{ICC}$ & 0,000395 & 11,250 & 4.807 & 0,000408 & 11,394 & 1.366 & 0,000389 & 12,676 & 18.971 \\
\hline $\mathrm{ICF}$ & 2,118 & 6,831 & 4.814 & 2,098 & 6,798 & 1.367 & 2,173 & 7,641 & 18.981 \\
\hline ICP1 & 3,124 & 10,594 & 4.814 & 3,056 & 10,397 & 1.367 & 3,191 & 11,890 & 18.981 \\
\hline ICP2 & 8,305 & 10,084 & 4.814 & 8,391 & 10,490 & 1.367 & 8,997 & 10,858 & 18.891 \\
\hline
\end{tabular}

${ }^{1} \mathrm{PCA}=$ peso calculado aproximado $(\mathrm{kg}) ; \mathrm{VSE}=$ vazio subesternal $(\mathrm{m}) ; \mathrm{IP}=$ índice peitoral $(\mathrm{m}) ; \mathrm{IC}=$ índice de compacidade; IDT = índice dactilotorácico; ICC = índice de carga na canela $(\mathrm{m} / \mathrm{kg}) ; \mathrm{ICF}=$ índice de conformação; ICP1 = índice de compacidade 1 ; ICP2 = índice de compacidade $2 .{ }^{2} \bar{X}$ : média; CV \%: coeficiente de variação em porcentagem; n: número de animais avaliados. 
Observa-se que os garanhões apresentaram valor médio, para peso calculado aproximado (PCA), superior tanto às fêmeas quanto aos machos castrados (Tab. 1 e 2). O maior valor encontrado para esses pode ser explicado pela fórmula utilizada para o cálculo da estimativa do peso, pois considera a medida do perímetro torácico, que, por sua vez, é influenciada pelas práticas nutricionais, bem como o maior vigor físico e maiores medidas de altura, observados nos machos (McManus et al., 2005). Além disso, ambas as variáveis foram influenciadas pelo sexo (Tab. 2).
As observações acerca da proporcionalidade dos garanhões, fêmeas e machos castrados da raça Campolina foram obtidas mediante os índices apresentados na Tab. 1. O vazio subesternal (VSE) pode ser definido como a distância compreendida entre a região do esterno e o solo. O VSE apresenta relação estreita com o comprimento de membros e a altura dos costados, influenciando, dessa forma, na estabilidade do equino pelo consequente posicionamento do centro de gravidade. Observa-se que os garanhões e os castrados apresentaram, para a medida do VSE, média maior que as fêmeas.

Tabela 2. Resultados de p-valores para a comparação dos índices morfométricos entre categorias de sexo e faixa etária de animais da raça Campolina obtidos por análise de variância (A) ou teste de KruskalWallis $(\mathrm{K})$.

\begin{tabular}{llccc}
\hline \multirow{2}{*}{ Índices $^{1}$} & Sexo & \multicolumn{3}{c}{ Idade } \\
\cline { 2 - 4 } & & Garanhões & Machos castrados & Fêmeas \\
\hline PCA & $0,000^{* *}(\mathrm{~K})$ & $0,050^{*}(\mathrm{~A})$ & $0,010^{*}(\mathrm{~A})$ & $0,000^{* *}(\mathrm{~K})$ \\
VSE & $0,000^{* *}(\mathrm{~K})$ & $0,000^{* *}(\mathrm{~A})$ & $0,000^{* *}(\mathrm{~K})$ & $0,000^{* *}(\mathrm{~K})$ \\
IP & $0,000^{* *}(\mathrm{~K})$ & $0,001(\mathrm{~K})$ & $0,000^{* *}(\mathrm{~A})$ & $0,000^{* *}(\mathrm{~K})$ \\
IC & $0,000^{* *}(\mathrm{~K})$ & $0,000^{* *}(\mathrm{~A})$ & $0,043^{*}(\mathrm{~A})$ & $0,000^{* *}(\mathrm{~K})$ \\
IDT & $0,000^{* *}(\mathrm{~K})$ & $0,562^{\mathrm{ns}}(\mathrm{A})$ & $0,781^{\mathrm{ns}}(\mathrm{A})$ & $0,098^{\mathrm{ns}}(\mathrm{K})$ \\
ICC & $0,000^{* *}(\mathrm{~K})$ & $0,072^{\mathrm{ns}}(\mathrm{A})$ & $0,214^{\mathrm{ns}}(\mathrm{A})$ & $0,000^{* *}(\mathrm{~K})$ \\
ICF & $0,000^{* *}(\mathrm{~K})$ & $0,925^{\mathrm{ns}}(\mathrm{A})$ & $0,003^{* *}(\mathrm{~A})$ & $0,000^{* *}(\mathrm{~K})$ \\
ICP1 & $0,000^{* *}(\mathrm{~K})$ & $0,529^{\mathrm{ns}}(\mathrm{A})$ & $0,004^{* *}(\mathrm{~A})$ & $0,000^{* *}(\mathrm{~K})$ \\
ICP2 & $0,000^{* *}(\mathrm{~K})$ & $0,307^{\mathrm{ns}}(\mathrm{A})$ & $0,004^{* *}(\mathrm{~A})$ & $0,000^{* *}(\mathrm{~K})$ \\
\hline
\end{tabular}

${ }^{1} \mathrm{PCA}=$ peso calculado aproximado $(\mathrm{kg}) ; \mathrm{VSE}=$ vazio subesternal $(\mathrm{m}) ; \mathrm{IP}=$ índice peitoral $(\mathrm{m})$; IC $=$ índice de compacidade; IDT = índice dactilotorácico; ICC = índice de carga na canela $(\mathrm{m} / \mathrm{kg}) ; \mathrm{ICF}=$ índice de conformação; ICP1 = índice de compacidade $1 ;$ ICP2 = índice de compacidade 2 . $* * \mathrm{P}<0,001 ; * \mathrm{P}<0,05$. ns: não significativo; (A) ANOVA; (K) Teste de Kruskal-Wallis.

Destaca-se que, independentemente do sexo, os animais foram classificados, segundo o índice peitoral (IP), como longe do chão. Os garanhões são mais distantes do solo em relação aos machos castrados e estes em relação às fêmeas. Quanto aos machos castrados, o IP (-0,204m) evidencia que eles são, em média, melhor relacionados com o solo que os garanhões. $\mathrm{O}$ valor de IP encontrado para os garanhões demonstra que a média deste estudo é um pouco maior em relação àquela encontrada por Berbari Neto (2009) também para a raça Campolina, o qual verificou valor $-0,296 \mathrm{~m}$. O valor médio do IP para as fêmeas demonstra que elas apresentaram-se um pouco mais longe do chão, se comparadas ao observado por Campos
(2006), quando a autora encontrou valor para este índice de $-0,1692 \mathrm{~m}$. McManus et al. (2008), ao avaliarem machos e fêmeas da raça Pantaneiro, embora os tenham classificado como "longe do chão", verificaram que as fêmeas $(0,584)$ são mais longe do chão que os machos $(0,572)$.

Com base no índice corporal (IC), os garanhões e machos castrados foram classificados como mediolíneos (Tab. 1). Esse resultado corrobora o de Berbari Neto (2009), o qual encontrou em seu estudo, com os garanhões da raça, o valor do IC de 0,879 e sugeriu uma tendência de crescimento para esse índice baseado nas observações médias dos últimos anos do seu 
estudo. Em relação às fêmeas, estas foram classificadas como brevilíneas, não compreendendo a faixa de classificação recomendada pelo padrão da raça, que estabelece como ideal animais mediolíneos (Inglês et al., 2004). Contudo, existem boas perspectivas de enquadramento destas ao recomendado pelo padrão racial, uma vez que Campos (2006) encontrou valor para o IC de 0,85693, com tendência de aumento. Esses resultados podem ser um indicativo do empirismo utilizado na seleção, durante muito tempo, por grande parte dos criadores da raça, uma vez que os animais castrados apresentaram, em média, IC mais adequado que fêmeas e garanhões. Para cavalos Quarto de Milha, Rezende et al. (2015) obtiveram média de 0,86, que compreende o limite para classificação como mediolíneos.

O índice dactilotorácico (IDT) permite averiguar se a capacidade óssea do animal é condizente com seu peso corpóreo. Observa-se, na Tab. 3, que os garanhões e os machos castrados apresentaram valor médio que os classifica como eumétricos, atendendo, dessa forma, às sugestões do padrão da raça (Inglês et al., 2004). Diferentemente dos valores médios do IDT observados para os garanhões e castrados, as fêmeas apresentaram para esse índice média de 0,103, classificando-as como hipométricas. Entretanto, essa média apresenta-se maior que a relatada por Campos (2006), que, embora tenha classificado as fêmeas da raça Campolina como hipométricas, constatou tendência de aumento para esse índice, corroborando os resultados do presente estudo. Assim como no presente estudo (Tab. 2), em animais da raça Pantaneiro, o sexo influenciou o IDT, para o qual os machos apresentaram média superior às fêmeas (MacManus et al., 2008). Em animais da raça Mangalarga Machador, Cabral et al. (2004) verificaram que, ao nascimento, tanto fêmeas quanto machos foram classificados como hipermétricos até os 12 meses de idade. Entretanto, à idade adulta, para ambos os sexos os animais foram classificados como eumétricos.

O índice de carga na canela (ICC) permite avaliar a capacidade dos membros de deslocar a massa corporal. De acordo com esse índice, valores muito baixos podem significar membros fracos (Oom e Ferreira, 1987). Nos valores médios de ICC, os machos castrados apresentaram, em média, membros mais fortes que os garanhões e estes em relação às fêmeas (Tab. 1). O valor encontrado para os garanhões é muito próximo ao observado por Berbari Neto (2005). Para as fêmeas, a média observada é um pouco menor que a encontrada por Campos (2006), que relatou o valor de 0,00039 para o referido índice. Cabral et al. (2004) encontraram, em fêmeas da raça Mangalarga Marchador, o valor do ICC de 0,0005, denotando, na média, fêmeas com membros mais fortes em relação ao seu peso corporal que as da raça Campolina.

O índice de conformação (ICF) exprime a relação entre o perímetro torácico e a altura. Segundo McManus et al. (2005), cavalos do tipo sela devem apresentar ICF igual a 2,1125, enquanto valores acima deste indicam animais aptos à tração. A média dos garanhões $(2,118)$ apresentou valor muito próximo ao recomendado. Contudo, os valores médios observados para os machos castrados $(2,098)$ e as fêmeas $(2,173)$ apresentaram-se abaixo e acima do recomendado, respectivamente. McManus et al. (2005), em estudo com animais da raça Campeira, encontraram valores médios para o ICF de 2,092. Para animais da raça Quarto de Milha, utilizados em provas de laço comprido, Rezende et al. (2015) obtiveram ICF médio de 2,04, sugerindo-os como aptos apenas para trabalho com sela a tração leve.

O índice de compacidade (ICP) tem por finalidade identificar a aptidão do animal por meio da relação entre o seu peso e a altura de cernelha. Para o ICP1, o valor médio encontrado para as fêmeas permitiu classificá-las como de tração pesada, com valor superior aos 3,15, recomendado para animais com essa aptidão (Tab. 1). O valor encontrado para a média do ICP1 dos machos castrados e dos garanhões os classificou como animais intermediários entre a tração ligeira e a tração pesada, sendo a média observada para os garanhões bem próxima ao valor limite entre as duas classificações. McManus et al. (2005) encontraram, para os animais da raça Campeira, valor médio de ICP1 $(2,915)$ maior que o recomendado para os animais tipo sela.

O ICP2, que considera as mesmas medidas lineares e classificações de aptidão do ICP1, 
difere deste pelos valores de classificação. Os valores médios do ICP2 (Tab. 1) de garanhões, machos castrados e fêmeas, classificaram-nos como aptos à tração ligeira, diferentemente do observado no ICP1, quando a média encontrada para as fêmeas classificava-as como aptas à tração pesada. McManus et al. (2005) encontraram valor médio de 9,558 para os animais da raça Campeira, classificando-os como aptos à tração pesada, diferindo, assim, da classificação gerada pelo ICP1 no mesmo estudo. Por outro lado, os resultados apresentados por Rezende et al. (2015) demonstraram que tanto o ICP1 $(2,84)$ quanto o ICP2 $(8,89)$ classificaram os equinos Quarto de Milha como aptos à tração ligeira. Além das divergências encontradas entre as duas fórmulas utilizadas para esse índice, os estudos evidenciaram que, de forma geral, os animais das diferentes raças avaliadas tiveram suas classificações superestimadas de acordo com seus respectivos padrões. Este é um indicativo para que mais estudos sejam realizados com a finalidade de melhorar a classificação gerada por tal índice.
Com base nos resultados da análise de variância, apresentados na Tab. 2, verificou-se que o sexo influenciou todos os índices morfométricos ( $p$ valor $\leq 0,001)$. Embora a funcionalidade da raça Campolina não faça distinção entre machos e fêmeas, esse resultado pode ser explicado pela diferença na altura mínima, entre os sexos, determinada pelo padrão racial. Conforme verificado por Lucena et al. (2015), a altura da cernelha (ACER) é fortemente correlacionada com várias medidas lineares. Além disso, esses resultados são confirmados pela análise da Tab. 3, na qual se observa que ACER é significativamente correlacionada com todos os índices do presente estudo. McManus et al. (2005), ao avaliarem medidas lineares e índices morfométricos em equinos da raça Campeiro, observaram que o sexo influenciou o perímetro torácico (PTOR), o qual foi maior em fêmeas. Esse resultado vai de encontro aos obtidos no presente estudo, para o qual os garanhões apresentaram PTOR maior, tanto em relação aos machos castrados quanto às fêmeas (Tab. 1).

Tabela 3. Correlação entre medidas lineares e índices morfométricos de garanhões, machos castrados e fêmeas da raça Campolina

\begin{tabular}{|c|c|c|c|c|c|c|c|c|c|}
\hline $\begin{array}{l}\text { Medidas } \\
\text { Lineares }^{1} \\
\text { Índices }^{2} \\
\end{array}$ & / PCA & VSE & IP & IC & IDT & ICC & ICF & ICP1 & ICP2 \\
\hline ACER & $0,474 * *$ & $0,741 * *$ & $-0,333 * *$ & $0,297 * *$ & $0,025 * *$ & $-0,320 * *$ & $0,066 * *$ & $0,218 * *$ & $-0,328 * *$ \\
\hline ADOR & $0,450 * *$ & $0,812 * *$ & $-0,392 * *$ & $0,264 * *$ & $0,022 * *$ & $-0,304 * *$ & $0,066 * *$ & $0,209 * *$ & $-0,305 * *$ \\
\hline $\operatorname{ACOS}$ & $0,526 * *$ & $-0,130 * *$ & $0,625^{* *}$ & $-0,044 * *$ & $-0,104 * *$ & $-0,413 * *$ & $0,369 * *$ & $0,436^{* *}$ & $0,162 * *$ \\
\hline CCOR & $0,481 * *$ & $0,506^{* *}$ & $-0,140 * *$ & $0,482 * *$ & $0,054 * *$ & $-0,311 * *$ & $0,168 * *$ & $0,287 * *$ & $-0,153 * *$ \\
\hline PTOR & $0,997 * *$ & $0,159 * *$ & $0,149 * *$ & $-0,533 * *$ & $-0,394 * *$ & $-0,889 * *$ & $0,909 * *$ & $0,960 * *$ & $0,671 * *$ \\
\hline PCAN & $0,340 * *$ & $0,214 * *$ & $-0,020 * *$ & $0,057 * *$ & $0,726 * *$ & $0,102 * *$ & $0,209 * *$ & $0,261 * *$ & $0,050 * *$ \\
\hline
\end{tabular}

${ }^{1}$ ACER $=$ altura de cernelha $(\mathrm{m})$; ADOR $=$ altura de dorso $(\mathrm{m})$; ACOS = altura de costados $(\mathrm{m})$; CCOR = comprimento do corpo $(\mathrm{m}) ; \quad$ PTOR = perímetro torácico $(\mathrm{m}) ;$ PCAN = perímetro de canela $(\mathrm{m}) .{ }^{2} \mathrm{PCA}=$ peso calculado aproximado $(\mathrm{kg})$; VSE $=$ vazio subesternal $(\mathrm{m})$; IP $=$ índice peitoral $(\mathrm{m})$; IC $=$ índice de compacidade; IDT = índice dactilotorácico; ICC = índice de carga na canela $(\mathrm{m} / \mathrm{kg}) ; \mathrm{ICF}=$ índice de conformação; ICP1 = índice de compacidade $1 ; \mathrm{ICP} 2=$ índice de compacidade $2 .{ }^{*} * \mathrm{P}<0,001 ;{ }^{*} \mathrm{P}<0,05$.

As faixas etárias, testadas individualmente para cada categoria (garanhões, machos castrados e fêmeas), influenciaram vários índices. Para os garanhões, apenas o peso calculado aproximado, vazio subesternal e índice corporal sofreram efeito significativo ( $\mathrm{p}$-valor $\leq 0,05$ ), enquanto para as fêmeas todos os índices foram afetados ( $p$-valor $\leq 0,001$ ), exceto o índice dactilotorácico
$($ IDT) $(\mathrm{p}$-valor $=0,098)$. Do mesmo modo, para os machos castrados, excetuando-se o IDT e o índice de carga na canela (ICC), os demais índices foram também influenciados ( $p$-valor $\leq 0,05)$. Zamborlini et al. (1996), ao estimarem fatores ambientais e parâmetros genéticos em equinos da raça Mangalarga Marchador, destacaram que a idade à avaliação, o sexo e o 
ano influenciaram significativamente a maioria das medidas avaliadas e devem ser consideradas nos casos de estimação do mérito genético dos animais. Para animais da raça Pantaneiro, MacManus et al. (2008) verificaram que o sexo foi significante para a maioria dos índices, ao passo que a idade influenciou apenas o índice corporal relativo, os índices peitoral e dactilotorácico.

Todas as medidas lineares utilizadas apresentaram correlação significativa com os índices obtidos (Tab. 3). Uma vez que a maioria dessas medidas apresentam forte correlação entre si (Lucena et al., 2015), aquelas que são influenciadas pela idade afetam diretamente seus pares correlatos e, por consequência, os índices morfométricos derivados delas. Miserani et al. (2002) verificaram que as medidas altura de costados e comprimento corpóreo, entre outras, foram influenciadas pela idade em animais da raça Pantaneiro.

\section{CONCLUSÕES}

Conclui-se que o sexo influenciou todas as variáveis analisadas, assim como a idade apresentou maior efeito para as fêmeas e os machos castrados do que para os garanhões. Os machos da raça Campolina, registrados em definitivo na ABCCCampolina, devem ser considerados "longe do chão", mediolíneos e eumétricos, tendo os machos castrados maior capacidade de carga nos membros que os garanhões. As fêmeas foram classificadas como "longe do chão", brevilíneas e hipométricas, apresentando menor capacidade de carga nos membros do que as demais categorias. A classificação funcional com base nos índices de compacidade gerou resultados diferentes, indicando que mais estudos devem ser feitos a fim de melhorar a classificação gerada por esse índice.

\section{REFERÊNCIAS}

BERBARI NETO, F. Evolução de medidas lineares e avaliação de índices morfométricos em garanhões da raça Campolina. 2005. $107 f$. Dissertação (Mestrado em Produção Animal) Universidade Estadual do Norte Fluminense. Campo dos Goytacazes, RJ.
BERBARI NETO, F. Morfometria de campeões e não-campeões na raça Campolina. 2009. 105f. Tese (Doutorado em Ciência Animal) Universidade Estadual do Norte Fluminense. Campo dos Goytacazes, RJ.

BRETAS, M.S.; BERGMANN, J.A.G.; PROCÓPIO, A.M. Descrição dos pontos anatômicos para a tomada de medidas lineares e angulares nos equinos da raça Mangalarga Marchador. In: SEMANA DE INICIAÇÃO CIENTÍFICA, 2003, Belo Horizonte, MG. Anais... Belo Horizonte: UFMG, 2003. Cd Rom. (Resumo).

BUSSAB, W.O.; MORETTIN, P. Estatística básica. 8.ed. São Paulo: Saraiva, 2013.

CABRAL, G.C.; ALMEIDA, F.Q.; QUIRINO, C.R. et al. Avaliação morfométrica de equinos da raça Mangalarga Marchador: índices de conformação e proporções corporais. Rev. Bras. Zootec., v.33, Supl.1, p.1798-1805, 2004.

CAMPOS, C.C. Evolução de medidas lineares $e$ avaliação de índices morfométricos em fêmeas da raça Campolina. 2006. 63f. Dissertação (Mestrado em Produção Animal) - Universidade Estadual do Norte Fluminense, Campo dos Goytacazes, RJ.

COSTA, M.D.; BERGMANN, J.A.G.; PEREIRA, C.S. et al. Caracterização das proporções morfométricas dos pôneis da raça Brasileira. Arq. Bras. Med. Vet. Zootec., v.50, p.455-460, 1998.

INGLÊS, F.P.L.D; $\quad$ VIANNA, S.A.B.; PROCÓPIO, A.M. Padrão racial comentado do cavalo Campolina. Belo Horizonte. ABCCC. 2004. 6p.

LESBRE, F.X. Précis d'exterieur du cheval et des principanx domestiques. Paris. Vigot Frères, 1920. 500p.

LEVENE, H. Robust test for equality of variances. In: OLKIN, I.; SGHURYE, S.G.; HOEFFDING, W. et al. (Eds.). . Contributions to probability and statistics: essays in honor of Harold Hotteling. Palo Alto: Stanford University, 1960. p.279-292.

LUCENA, J.E.C.; VIANNA, S.A.B.; BERBARI NETO, F. et al. Estudo comparativo das proporções morfométricas entre garanhões e castrados da raça Campolina. Semina: Cienc. Agr., v.36, p.353-366, 2015. 
MCMANUS, C.M.; SANTOS, S.A.; SILVA, J.A. et al. Body indices for the pantaneiro horse. Braz. J. Vet. Res. Anim. Sci., v.45, p.362-370, 2008

MCMANUS, C.; FALCÃO, R.A.; SPRITZE, A. et al. Caracterização morfológica de equinos da raça Campeiro. Rev. Bras. Zootec., v.34, p.15531562, 2005.

MISERANI, M.G.; MCMANUS, C.; SANTOS, S.A. et al. Avaliação dos fatores que influem nas medidas lineares do cavalo Pantaneiro. Rev. Bras. Zootec., v.31, p.335-341, 2002.

OOM, M.M.; FERREIRA, J.C. Estudo biométrico do cavalo Alter. Rev. Port. Ciênc. Vet., v.82, p.101-148, 1987.

REGULAMENTO do serviço de registro genealógico e padrão racial. Belo Horizonte, MG: ABCCC, 1995.18p.

RESENDE, G.R.O. Origem do cavalo Campolina. In: CONVENÇÃO NACIONAL DO CAVALO CAMPOLINA, 3. Abril de 1979; Belo Horizonte. Rev. Cavalo Campolina. Ano 1, n. ${ }^{\circ} 1$. 1979.
REZENDE, M.P.G.; ABREU, U.G.P.; SOUZA, J.C. et al. Morfologia corporal de equinos Quarto de Milha puros e mestiços utilizados no Laço Comprido no Mato Grosso do Sul. Arch. Zootec. v.64, p.183-186. 2015.

RIBEIRO, D.B. O cavalo: raças, qualidades e defeitos. 2.ed. São Paulo: Editora Globo. 1989. $318 \mathrm{p}$.

TORRES, A.D.P.; JARDIM, W.R. Criação do cavalo e de outros equinos. São Paulo: Editora Nobel. 1992. 654p.

ZAMBORLINI, L.C.; BERGMANN, J.A.G.; PEREIRA, C.S. et al. Estudo genéticoquantitativo de medidas lineares de equinos da raça Mangalarga Marchador - I. Estimativas dos fatores de ambiente e parâmetros genéticos. Rev. Bras. Ciênc. Vet., v.3, p.33-37. 1996. 